GASTROINTESTINAL INFLAMMATION

\title{
CXCR3 and $\alpha_{E} \beta_{7}$ integrin identify a subset of CD8+ mature thymocytes that share phenotypic and functional properties with CD8+ gut intraepithelial lymphocytes
}

\author{
F Annunziato, L Cosmi, F Liotta, E Lazzeri, P Romagnani, R Angeli, L Lasagni, \\ R Manetti, F Marra, C Gerard, I Petrai, P Dello Sbarba, F Tonelli, E Maggi, \\ S Romagnani
}

See end of article for authors' affiliations

Correspondence to: Professor S Romagnani, Dipartimento di Medicina Interna, Universitò di Firenze, Viale Morgagni 85 , Firenze 50134 Italy; s.romagnani@ dmi.unifi.it

Revised version received 8 November 2005 Accepted for publication 22 November 2005 Published online first 20 December 2005
Background: We previously demonstrated the existence of two distinct subsets of $\mathrm{T}$ cell receptor (TCR) $\alpha \beta+C D 8 \alpha \beta+$ single positive (SP) cells in human postnatal thymus which express the chemokine receptor CCR7 or CXCR3 and migrate in vitro in response to their specific ligands.

Aim: To investigate whether these two CD8+ thymocyte subsets had distinct peripheral colonisation.

Methods: TCR $\alpha \beta+C D 8+$ SP cells were obtained from normal postnatal thymus, mesenteric lymph node (LNs), small bowel, and peripheral blood (PB) specimens. Cells were then evaluated for expression of surface molecules, cytolytic potential, telomere length, and profile of cytokine production.

Results: CD8+CCR7+CXCR3 - thymocytes exhibited CD62L, in common with those which localise to LNs. In contrast, CD8+CCR7-CXCR3+ thymocytes lacked CD62L but exhibited CD103, similar to intraepithelial lymphocytes (IELs) present in the gut mucosa where the CXCR3 ligand, CXCL10, and the CD103 ligand, E-cadherin, are highly and consistently expressed. In addition, thymocytes and gut CD8+CXCR3+CD103+ cells showed comparable telomere length, which was higher than that of PB CXCR3+CD8+ T cells. However, both of these populations contained perforin and granzyme A, and displayed the ability to produce interferon $\gamma$ and interleukin 2. Of note, CXCR3 deficient, in comparison with wild-type C57Black/6, mice showed decreased proportions of CD3+CD8 $\alpha \beta+$ and increased proportions of $C D 3+C D 8 \alpha \alpha+$ lymphocytes at gut level. Moreover, adoptive transfer of CD3+CD8 $\alpha \beta+$ thymocytes from wild-type into CXCR3 deficient mice resulted in a significant increase in CD3+CD8 $\alpha \beta+$ $T$ cells in the gut mucosa but not in other tissues.

Conclusions: The results of this study demonstrate the existence of a previously unrecognised subset of $\mathrm{TCR} \alpha \beta+\mathrm{CD} 8 \alpha \beta+\mathrm{SP} C X C R 3+\mathrm{CD} 103+$ thymocytes which share phenotypic and functional features with CD8+ IELs, thus suggesting the possibility of their direct colonisation of the gut mucosa. naïve T cells) and CXCR4 (broadly expressed), both of which also bind homeostatic chemokines. Thus far, a role for CCR9 in the entry of $\mathrm{T}$ cells into lymph nodes (LNs) has not been demonstrated. The ligand for CCR9 is CCL25, which is selectively expressed in the small intestine, ${ }^{13}{ }^{14}$ raising the possibility that this chemokine is involved in the trafficking of naïve CCR9+ T cells to lymphoid structures of the small intestine. However, it is also possible that the presence of CCR9 in naïve T cells is simply a remnant of development in the thymus, as CCR9 is broadly expressed by thymocytes..$^{13} 15$ In contrast, CXCR4 does appear to contribute to T cell entry into LNs. Indeed, CXCL12 (the ligand for CXCR4) has been shown to promote transendothelial migration of $\mathrm{T}$ cells across HEVs. ${ }^{16}$ Hence CCR7 and CXCR4 may cooperate in the task of naïve $\mathrm{T}$ cell recruitment into LNs. Of note, other chemokine receptors with expression on naïve $\mathrm{T}$ cells have not yet been identified. CXCR3 binds the chemokines CXCL10, CXCL9, and CXCLll, is expressed on the surface of different cell types, including activated $\mathrm{T}$ cells and natural killer cells, and

Abbreviations: SP, single positive; $\mathrm{PB}$, peripheral blood; IELs, intraepithelial lymphocytes; HEV, high endothelial venules; mAbs, monoclonal antibodies; LNs, lymph nodes; TCR, T cell receptor; IL, interleukin; IFN- $\gamma$, interferon $\gamma$; MNC, mononuclear cells; PBS, phosphate buffered saline 
subsets of inflammatory dendritic cells, macrophages, and B cells, and its expression on $\mathrm{T}$ lymphocytes is upregulated by inflammation. ${ }^{3}$

Recently, we discovered the presence in human postnatal thymus of a subset of T cell receptor (TCR) $\alpha \beta+C D 8 \alpha \beta+$ SP cells which express CXCR3 and migrate in vitro in response to CXCL10, CXCL9, and CXCL11, but not to CCL19 or CCL21. ${ }^{17}$ Thymic $\mathrm{TCR} \gamma \delta$ and $\mathrm{CD} 3-\mathrm{CD} 56+$ (natural killer-like) cells also showed similar behaviour. ${ }^{17}$ In this study, we hypothesised that the two CD8+ thymocyte subsets (CCR7+ or CXCR3+) have distinct peripheral colonisation. Therefore, we assessed the presence of CCR7 and CXCR3, as well as of other phenotypic and functional markers, in TCR $\alpha \beta+C D 8 \alpha \beta+$ SP thymocytes and compared the phenotype of these cells with that of $\mathrm{CD} 8 \alpha \beta+\mathrm{T}$ cells present in different tissues, such as LNs, peripheral blood (PB), and small bowel. The results showed that SP CD8+ thymocytes expressing CXCR3 represent a distinct population of cells that selectively colonise to the lymphoid structures of the small intestine.

\section{MATERIAL AND METHODS \\ Human specimens}

Normal postnatal thymus specimens were obtained from 11 children aged between five days and three years, and from one 42 year old male who underwent corrective cardiac surgery. Normal mesenteric LNs, uninvolved small bowel specimens, and PB lymphocytes were all obtained from 18 patients who underwent surgery because of colon cancer (eight patients) or Crohn's disease (10 patients). Additional PB samples were obtained from 13 healthy volunteers. All patients gave informed consent. The procedures followed in the study were in accordance with the ethical standards of the Regional Committee on Human Experimentation which approved the study.

\section{Animals}

CXCR3 deficient mice backcrossed 10 generations into the C57Black/6 background have been described previously. ${ }^{18}$ Wild-type C57Black/6 mice were used as controls. Mice were maintained under specific pathogen free conditions until use.

\section{Reagents}

Purified anti-CD8 $\alpha$, as well as the fluorochrome conjugated anti-CD3, anti-CD4, anti-CD62L, anti-CD103, anti-CD20, anti-TCR $\gamma \delta$, anti-interleukin (IL)-2, anti-IL-4, anti-IL-10, anti-IL-13, anti-interferon $\gamma($ IFN- $\gamma)$, anti-perforin, and anti-granzyme A monoclonal antibodies (mAbs) were purchased from Becton Dickinson (San Jose, California, USA); anti-CD8 $\beta$ and anti-E-cadherin mAbs were obtained by Beckman Coulter (Brea, California, USA). Goat antimouse IgG Abs conjugated with magnetic beads were obtained from Miltenyi Biotec (Bisley, Germany). Purified fluorochrome conjugated isotype control and fluorochrome conjugated anti-isotype mAbs were purchased from Southern Biotechnology Associates (Birmingham, Alabama, USA). Purified and PE conjugated anti-CXCR3, anti-CCR9, and anti-CCR7 mAbs were obtained from R\&D (Minneapolis, Minnesota, USA). Anti-CXCL10 rabbit polyclonal Ab was from Peprotech (London, UK). Fluorochrome conjugated antimouse $\mathrm{CD} 4, \mathrm{CD} 8 \alpha$, and $\mathrm{CD} 8 \beta$ mAbs were purchased from Serotec (Oxford, UK).

Preparation of cell suspensions and isolation of IELs Thymus fragments were gently passed through a stainless steel mesh (Medimachine; Becton Dickinson) to obtain single cell suspensions from which mononuclear cells (MNC) were separated by centrifugation on Ficoll-Hypaque gradient. Specific isolation of intraepithelial lymphocytes (IELs) was performed as follows: the epithelial layer was removed using phosphate buffered saline (PBS) calcium and magnesium free supplemented with $1 \mathrm{mM}$ EDTA and $1 \mathrm{mM}$ DTT (Sigma Chemical Co, St Louis, Missouri, USA). After continuous agitation for one hour at $37^{\circ} \mathrm{C}$, single cell suspension was pelleted from the supernatant and washed once with PBS supplemented with $5 \%$ fetal calf serum. MNC were then separated by centrifugation on Ficoll-Hypaque gradient.

\section{Positive selection of CD8 $+\mathrm{T}$ cells}

Positive selection of CD8+ T cells was performed by high gradient magnetic cell sorting, as described elsewhere. ${ }^{11}$ Briefly, PBMNC or CD4 depleted thymocytes were incubated with an anti-CD8 mAb, extensively washed, and then further incubated with a microbead conjugated goat antimouse polyclonal Ab (Milteny Biotec GmbH, Bergisch Gladbach, Germany). After washing, cells were separated on a $\mathrm{LS}^{+}$ column. Purity of recovered cells was consistently higher than $98 \%$.

\section{Adoptive cell transfer in mice}

Thymocytes obtained from five wild-type C57Black/6 mice were depleted of CD4+ T cells using a microbead conjugated anti-CD4 mAb (Milteny Biotec GmbH). CD4+ cell depleted thymocyte suspensions were then incubated with a microbead conjugated anti-CD8 mAb and, after washing, separated on a LS ${ }^{+}$column (Milteny Biotec GmbH). Purity of recovered cells was consistently higher than 98\%. The majority of CD8+ thymocytes (>98\%) were CD $3+\mathrm{TCR} \alpha \beta \mathrm{CD} 8 \alpha \beta$ cells. CD8+ cells were counted and $2 \times 10^{7}$ cells were injected into the tail vein of three singenic CXCR3 deficient mice. After 24 hours, mice were sacrificed and gut, spleen, liver, and $\mathrm{PB}$ were monitored with flow cytometry for CD8 $\alpha \beta$ and CD8 $\alpha \alpha$ content.

\section{Flow cytometric analysis}

Flow cytometric analysis on cell suspensions was performed as detailed elsewhere. ${ }^{11}$ Assessment of cytoplasmic perforin and granzyme A expression was performed according to the manufacturer's instructions (BD Biosciences). Flow cytometric analysis of cytokines synthesis at the single cell level was performed as detailed elsewhere. ${ }^{19}$ Cells were analysed on a BDLSR cytometer using CellQuest software (BD Biosciences).

\section{Determination of telomere length}

Telomere staining was performed using the Dako Telomere PNA Kit/FITC, according to manufacturer's instructions (Dako, Glostrup, Denmark).

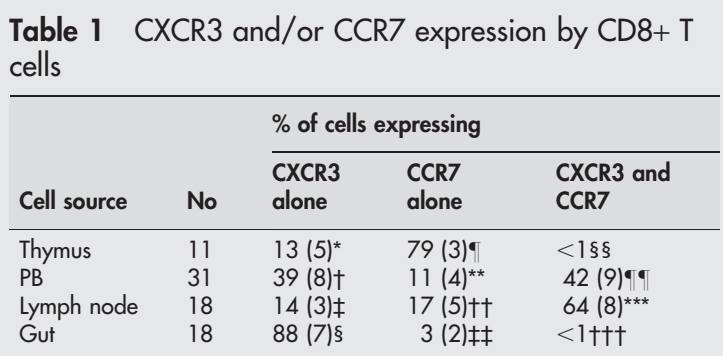

CXCR3 and CCR7 expression levels were assessed by flow cytometry in $\mathrm{CD} 3+\mathrm{CD} 8+$ gated cells.

Values are mean (SEM) of positive cells. $\mathrm{PB}$, peripheral blood.

${ }^{*} v \dagger, \mathrm{p}<0.05 ;{ }^{*} v \ddagger, N S ;{ }^{*} v \S, \mathrm{p}<0.01 ; \dagger v \ddagger, \mathrm{p}<0.05 ; \dagger v \S, \mathrm{p}<0.01$; $\ddagger v \S, p<0.01 ; \uparrow v^{* *}, p<0.01 ; \uparrow v+1, p=0.01 ; \uparrow v \neq \neq, p<0.01 ; * *$

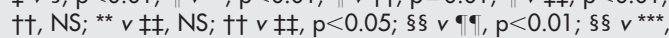

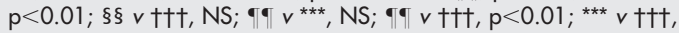
$p<0.01$. 


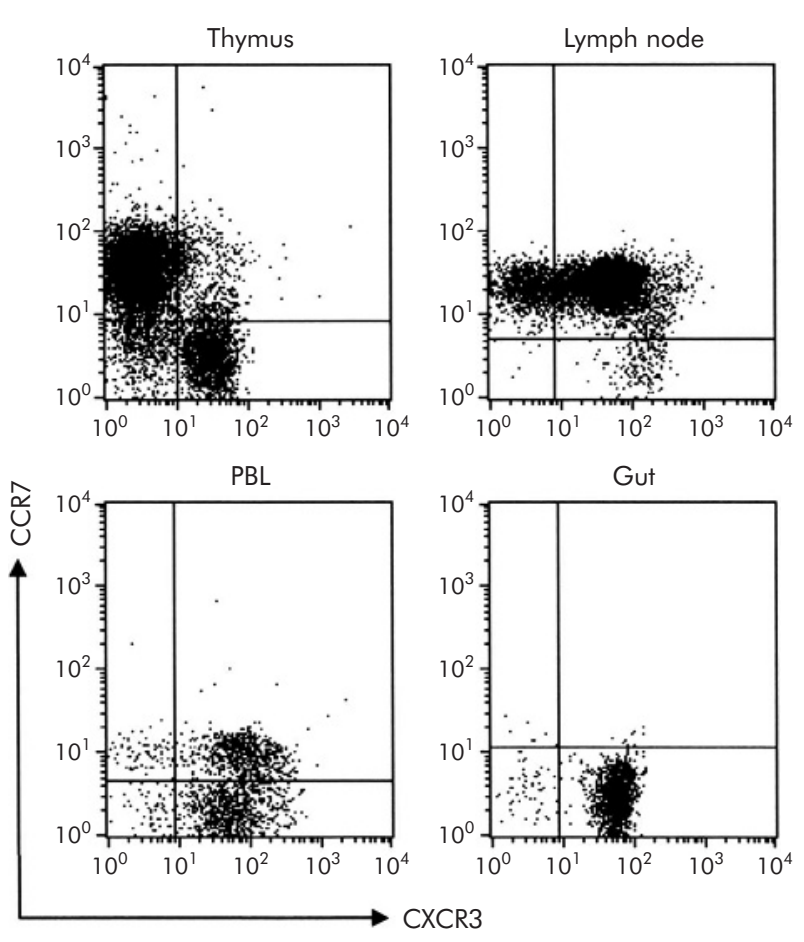

Figure 1 Different distribution of CXCR3 and CCR7 on T cell receptor (TCR) $\alpha \beta+C D 8+$ single positive T cells from thymus, lymph nodes, peripheral blood lymphocytes (PBL), and gut. Mononuclear cell (MNC) suspensions from tissue fragments and peripheral blood were obtained as described in material and methods. In the case of thymus derived MNC, CD4+CD8+ DP thymocytes were first removed by high gradient magnetic cell sorting, as described in material and methods. The remaining cells were then contemporaneously stained with anti-CCR7, anti-CXCR3, anti-CD3, and anti-CD8 fluorochrome conjugated monoclonal antibodies, and analysed on a BD-LSR cytometer. All plots represent $\mathrm{CD} 3+\mathrm{CD} 8+$ gated cells.

\section{Immunohistochemistry}

Immunohistochemical staining was performed on $10 \mu \mathrm{m}$ cryostat sections, fixed in $4 \%$ paraformaldehyde for 20 minutes, or in acetone for 10 minutes. Sections were subsequently exposed to $0.3 \%$ hydrogen peroxide-methanol solution to quench endogenous peroxidase activity. After 30 minutes of preincubation with normal horse serum (Vectastain ABC kit; Vector Laboratories, DBA, Milan, Italy), sections were layered for 30 minutes with antiCXCL10 $(10 \mu \mathrm{g} / \mathrm{ml})$ or anti-CK $(2 \mu \mathrm{g} / \mathrm{ml})$, anti-E-cadherin $(10 \mu \mathrm{g} / \mathrm{ml})$, anti-CXCR3 $(10 \mu \mathrm{g} / \mathrm{ml})$, or anti-CD8 $\beta$ chain $(10 \mu \mathrm{g} / \mathrm{ml})$ Abs, followed by biotinylated antimouse or antirabbit IgG $\mathrm{Ab}$, and the avidin-biotin-peroxidase complex (Vectastain ABC kit). 3-Amino-9-ethylcarbazole (AEC; Sigma) was used as a peroxidase substrate. Finally, sections were counterstained with Gill's haematoxylin and mounted with Kaiser's glycerol gelatin. All incubations were performed at room temperature. As negative control, primary Ab was replaced with an isotype matched $\mathrm{Ab}$ with irrelevant specificity. Double immunostaining was performed using the avidin-biotin-peroxidase system with two different substrates. To identify two different proteins on the same specimen, AEC (red colour) and Vector SG (bluish grey) substrates were used. After double immunostaining, sections were mounted with Kaiser's glycerol gelatin.

\section{Statistical analysis}

Statistical analysis was performed using the Student's $t$ test and the ANOVA test.
RESULTS

Analysis of CXCR3 and CCR7 expression by TCR $\alpha \beta+C D 8+$ SP T cells in human thymus and in other tissues

In a previous study, we demonstrated that TCR $\alpha \beta+C D 8+$ SP cells present in postnatal human thymus, unlike TCR $\alpha \beta C D 4+$ SP cells which migrate only in response to CCL19 or CCL21, could migrate also in response to CXCL10, CXCL9, or CXCL11. ${ }^{17}$ Virtually all of these cells expressed the CD $8 \alpha \beta$ heterodimer. ${ }^{17}$ To establish whether $\mathrm{CD} 8 \alpha \beta+$ thymocytes responding to CCL19 and CCL21 or to CXCL10, CXCL9, and CXCL11 were distinct or overlapping subsets, expression of the corresponding receptors, CCR7 and CXCR3, was contemporaneously assessed on TCR $\alpha \beta+C D 8 \alpha \beta+$ SP thymocytes from 11 postnatal human thymuses. In all cases, CXCR3 appeared to be expressed by smaller subset than, but clearly distinct from, CD8 $\alpha \beta+$ SP thymocytes expressing CCR7 (table 1, fig 1). The same results were obtained when thymic specimens derived from one 42 year old subject were assessed (data not shown). We asked therefore whether a similar dichotomy could also be observed at the level of mature CD8+ $\mathrm{T}$ cells from different human tissues. In LNs, more than $80 \%$ of CD8+ T cells expressed CCR7 and the majority also CXCR3 (table 1, fig 1). In $\mathrm{PB}$, despite great variability at the individual level, there were three populations of CD8+ $\mathrm{T}$ cells, one of which expressed both CXCR3 and CCR7 and the other two CXCR3, or CCR7 alone (table 1, fig 1). In normal small bowel specimens from all 18 subjects examined, the vast majority $(>80 \%)$ of recovered $\mathrm{T}$ cells were $\mathrm{CD} 8+$ and all expressed the CD $8 \alpha \beta$ heterodimer (data not shown) and exhibited CXCR3, but no CCR7 (table 1, fig 1), as previously described. ${ }^{20}$

\section{CD8+CXCR3+ thymocytes have phenotypic and functional properties different from CD8+CCR7+ thymocytes}

To further investigate the meaning of the above mentioned findings, the two CD8+ thymocyte subsets (CXCR3+CCR7and CCR7+CXCR3-) were assessed for expression of other phenotypic and functional markers, such as CD62L, CD103, perforin, and granzyme A. Virtually all CXCR3+CD8+ thymocytes were CD62L- and part of them showed expression of both CDI03 ( $\sim 30 \%$ ) and perforin ( 28\%) (fig 2 ). Of note, more than $50 \%$ of perforin expressing cells also exhibited CD103 (fig 2, insert). In contrast, virtually all CCR7+CD8+ thymocytes were CD62L+ but they did not express CD103 or perforin (fig 2). We therefore assessed the same markers on CD8+ T cells from LNs, PB, and small bowel. As shown in fig 2, CD8+ T cells present in the normal gut showed a phenotype quite similar to CXCR3+CD8+ thymocytes (CXCR3+CD62L-CD103+), differing only because of a more pronounced presence of perforin, whereas virtually all CCR7+CD8+ T cells (both CXCR3+ and CXCR3-) from LNs exhibited high CD62L, but no CD103, and poor perforin expression (fig 2). CXCR3+CCR7+CD8+ T cells present in $\mathrm{PB}$ were $\mathrm{CD} 62 \mathrm{~L}+$, but $\mathrm{CD} 103-$, and showed no perforin expression. In contrast, CXCR3+CCR7- circulating CD8+ T cells were CD62L-, a small proportion of them $(\sim 1 \%)$ expressed CD103, whereas all exhibited strong perforin expression (fig 2). The distribution of granzyme A in the same tissues appeared to be quite similar to that of perforin (data not shown).

\section{CXCR3+CD8+ thymocytes share phenotypic and functional properties with gut IELs}

Expression of both CXCL10 and CXCR3 was then investigated in normal small bowel specimens using double staining Immunohistochemistry. High CXCL10 expression was observed at the level of intestinal epithelial cells (fig 3A, 

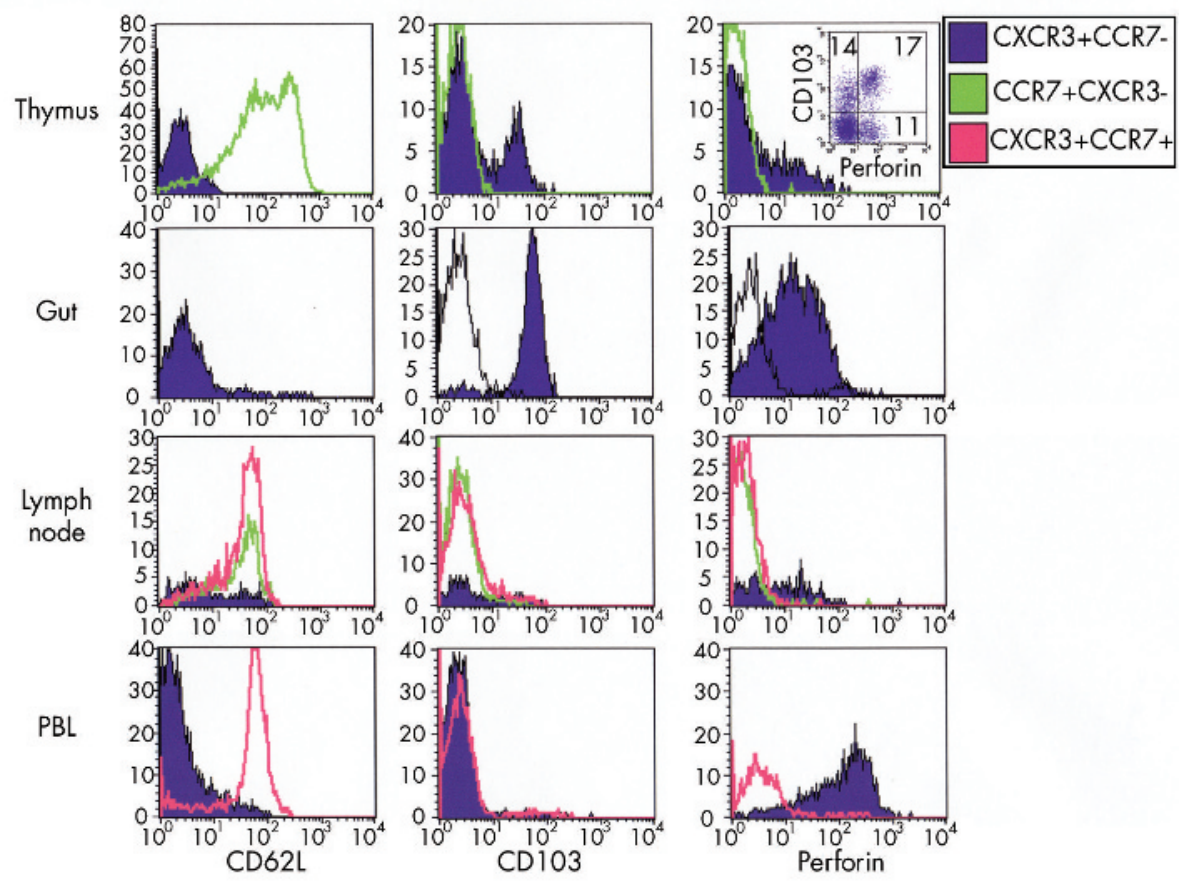

Figure 2 Assessment of CD62L, CD103, and perforin on CXCR3+CCR7-CD8+ and on CXCR3-CCR7+CD8+ cells. T cells from the thymus, gut, lymph node, and peripheral blood lymphocytes (PBL) were contemporaneously stained with anti-CD103 (or anti-CD62L, or anti-perforin), anti-CXCR3, anti-CCR7, and anti-CD8 fluorochrome conjugated monoclonal antibodies, and analysed on a BD-LSR cytometer. In the case of perforin staining, cells were previously fixed (for additional details, see material and methods). All histograms represent CD8+ gated cells. The insert plot was performed on CD8+ SP purified thymocytes (see material and methods) and stained for CXCR3, CCR7, CD103, and perforin.

3B) where it colocalised with E-cadherin (fig 3C). In the same tissue sections, high proportions of intraepithelial CD8+ $\mathrm{T}$ cells expressing CXCR3 were observed (fig 3D). As CXCL12 ${ }^{13}$ and CCL2 $5^{142122}$ have also been implicated in the chemoattraction of $\mathrm{T}$ cells to the gut mucosa, expression of the respective receptors, CXCR4 and CCR9, by CXCR3+CD8+ $\mathrm{T}$ cells was also investigated. Although a fraction of CXCR3+CD8+ gut $\mathrm{T}$ cells expressed CXCR4 (mean 43\%
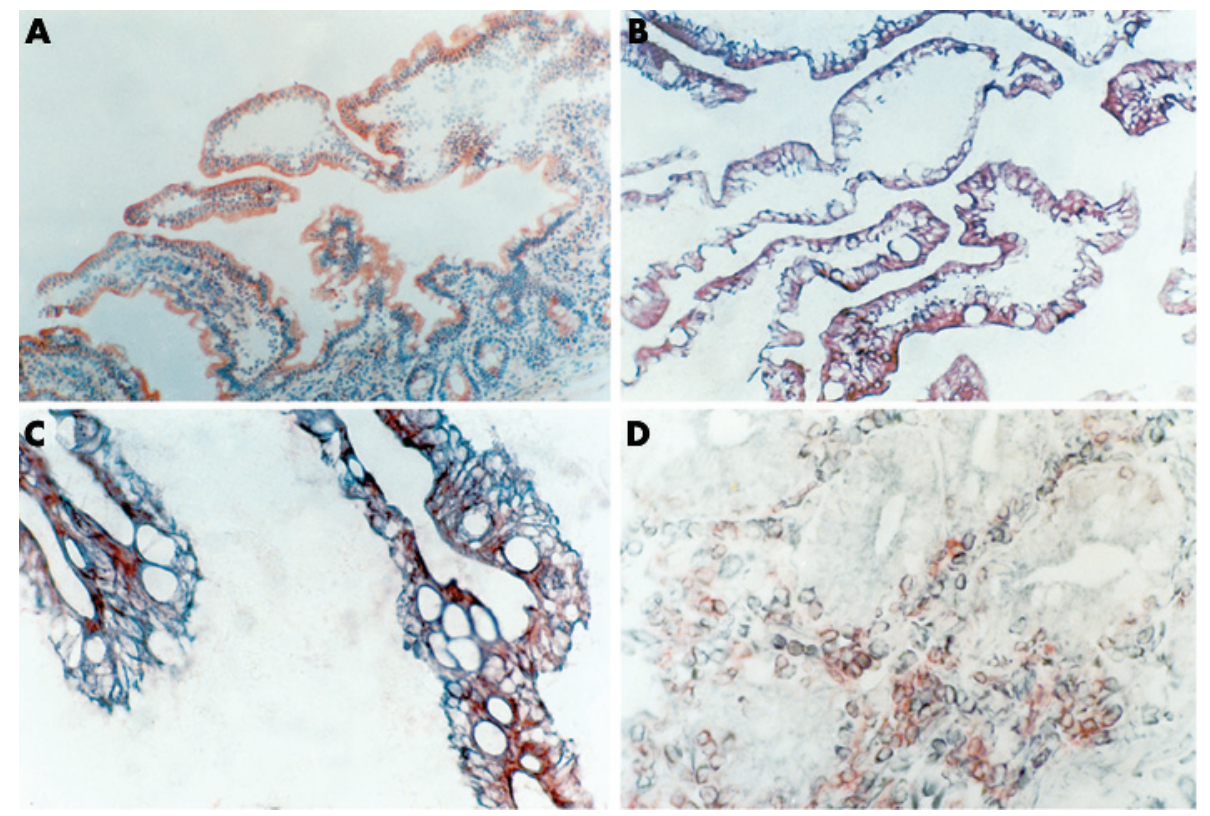

Figure 3 CXCL10 and E-cadherin colocalisation in intestinal epithelial cells and CXCR3 expression by intestinal T cell receptor (TCR) $\alpha \beta+C D 8+$ intraepithelial lymphocytes (IELs). (A) CXCL10 expression by intestinal epithelial cells. Sections of morphologically and histologically normal small bowel were stained with anti-CXCL10 polyclonal monoclonal antibody (red colour) and counterstained with Gyll's haematoxylin (blue colour) ( $\times 100)$. (B) Double label immunohistochemistry for CXCL10 (red colour) and CK (bluish-grey colour) showing CXCL10 expression by intestinal epithelial cells. No counterstain was applied $(\times 250)$. (C) Double label immunohistochemistry showing coexpression of CXCL10 (red colour) and E-cadherin (bluishgrey colour) in intestinal epithelial cells. No counterstain was applied $(\times 400)$. (D) Double label immunohistochemistry showing CXCR3 expression (bluish-grey colour) by CD $8 \alpha \beta+$ IELs (red colour). No counterstain was applied $(\times 400)$. 

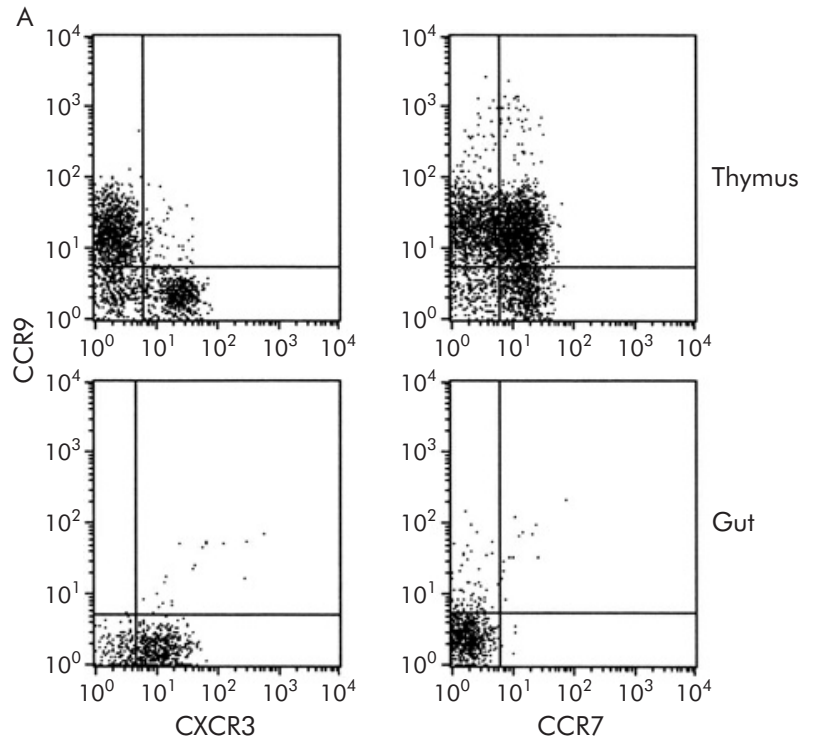

B
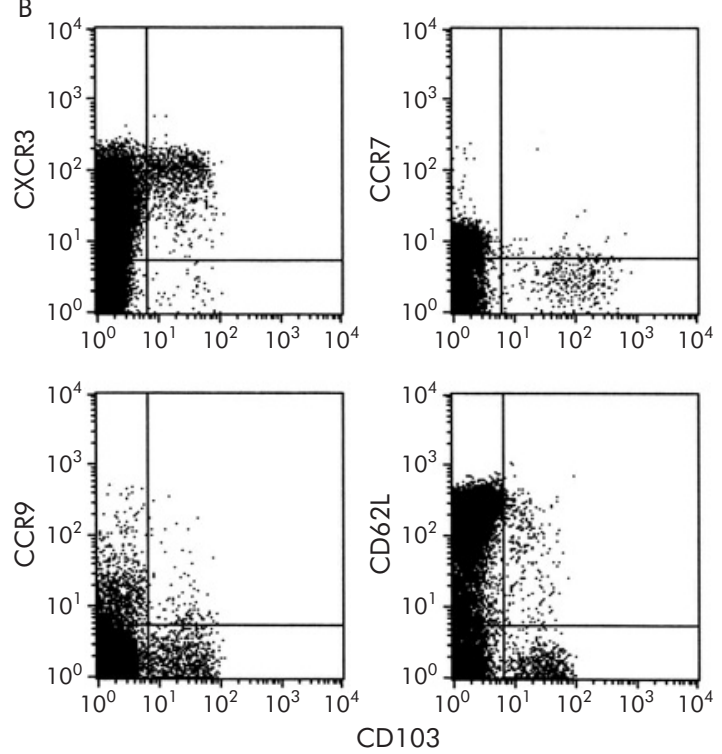

PBL

Figure 4 Assessment of CCR9 on thymus, gut, and peripheral blood (PBL) T cell receptor (TCR) $\alpha \beta+C D 8+T$ lymphocyte subsets. (A) Thymus and gut purified TCR $\alpha \beta+C D 8+T$ lymphocytes were contemporaneously stained with anti-CXCR3 or anti-CCR7 and anti-CCR9 monoclonal antibodies. (B) TCR $\alpha \beta+C D 8+P B$ T cells were assessed for expression of CD103 and CCR9, CXCR3, CCR7, or CD62L.

(SEM 8\%) in six bowel specimens analysed), the same receptor was also found in the majority (78\% (SEM 13\%) in 10 PB analysed) of PB CD8+ T cells, suggesting that this receptor was not critical for the selective homing of CXCR3+CD8+ lymphocytes to the gut. On the other side, CCR9 was found to be expressed in the thymus by the CCR7+, but not by the CXCR3+, CD8+ subset, and was virtually absent in CXCR3+CD8+ intestinal $\mathrm{T}$ cells (fig 4A). Accordingly, the small subset of CD103+CD8+ circulating $\mathrm{T}$ cells, which neither expressed CCR7 nor CD62L, also lacked CCR9 (fig 4B). In contrast, CCR9+ cells were detected in the CD20+ IEL population (data not shown).

Telomere length and profile of cytokine production of CXCR3+CD8+ cells from the thymus, gut, and PB were then compared. Analysis of telomere length of CXCR3+CD8+ thymocytes was comparable with that of both CCR7+CD8+ thymocytes and CXCR3+CD8+ gut IELs while telomere length of CXCR3+CD8+ PB lymphocytes was usually lower (fig 5A). Finally, both CXCR3+CD8+ thymocytes and CXCR3+CD8+ IELs showed the ability to produce both IL-2 and IFN- $\gamma$, but not IL-4, IL-10, or IL-13, in response to polyclonal stimulation whereas under the same experimental conditions CCR7+CD8+ thymocytes produced only small amounts of IL-2 (fig 5B). These findings suggest the possibility that CXCR3+CD8+ thymocytes represent a distinct subset able to directly migrate and colonise the gut mucosa.

\section{Reduced proportions of TCR $\alpha \beta+C D 8 \alpha \beta+T$ lymphocytes in the gut of CXCR3 gene deficient mice}

It is known that there are two major populations of CD3+CD8+ IELs in the mouse, one of which $(\mathrm{CD} 8 \alpha \alpha+)$ consists of TCR $\alpha \beta+$ and TCR $\gamma \delta+$ lymphocytes and the other $(\mathrm{CD} 8 \alpha \beta+)$ is represented only by TCR $\alpha \beta+$ lymphocytes. ${ }^{23}$

To provide further evidence that CXCR3 may play a role in the migration of $T C R \alpha \beta+C D 8 \alpha \beta+$ thymocytes to the gut, proportions of $\mathrm{CD} 3+\mathrm{CD} 8 \alpha \alpha+$ or $\mathrm{CD} 3+\mathrm{CD} 8 \alpha \beta+\mathrm{T}$ cells in the gut and PB of both CXCR3 gene deficient and wild-type mice were evaluated. There were no differences in the proportion of the two populations in the PB of CXCR3 deficient versus wild-type animals (fig 6A). In contrast, the proportions of $\mathrm{CD} 3+\mathrm{CD} 8 \alpha \alpha+\mathrm{T}$ cells were significantly increased whereas those of CD3+CD8 $\alpha \beta+\mathrm{T}$ cells were significantly reduced, in CXCR3 deficient in comparison with wild-type animals (fig 6B). CD3+CD8+ thymocytes (mainly composed of CD8 $\alpha \beta+$ cells) obtained from wild-type mice were then transferred to CXCR3 gene deficient mice, and after one day the proportions of $\mathrm{CD} 3+\mathrm{CD} 8 \alpha \beta+\mathrm{T}$ cells present in the gut, liver, spleen, and PB of these animals were compared with those present in untreated mice. As shown in fig 6C, there was a significant increase in $\mathrm{CD} 3+\mathrm{CD} 8 \alpha \beta+\mathrm{T}$ cells in the gut, but not in the $\mathrm{PB}$, spleen, or liver of adoptively transferred mice.

\section{DISCUSSION}

In this study, we demonstrated that TCR $\alpha \beta+C D 8 \alpha \beta+$ SP $\mathrm{T}$ cells expressing CXCR3 in human postnatal thymus were distinct from those expressing CCR7 and also exhibited different phenotypic and functional markers. CXCR3+CD8+ thymocytes lack CD62L, but express CD103, and part of them also possesses perforin and granzyme A. In contrast, CCR7+CD8+ thymocytes express CD62L, but lack CD103, perforin, and granzyme A. CD103, which is the $\alpha_{\mathrm{E}}$ chain of $\alpha_{\mathrm{E}} \beta_{7}$ integrin, has recently been found to define a distinct subset of naïve CD8+ T cells in the periphery, showing an age related decline in frequency which is significantly enhanced by thymectomy, thus suggesting their nature of recent thymic emigrants. $^{24}$ Of note, by assessing the same markers in different human tissues, we found that the great majority of $\mathrm{CD} 8+\mathrm{T}$ cells present in the small bowel showed a phenotype nearly identical to CXCR3+CD8+ thymocytes (CD62L-CD103+ perforin positive), whereas lymph nodes contained CD8+ T cells showing features more similar to the CCR7+CD8+ thymocyte subset (CD62L+CD103- perforin negative), although part of them expressed both CXCR3 and CCR7.

Based on these data we hypothesise that the two CD8+ thymocyte subsets (CXCR3+ or CCR7+) may have a different destiny and, therefore, also a different functional meaning. CCR7 expressing CD8+ thymocytes certainly colonise LNs through HEV. Coexpression of CXCR3 and CCR7 on a proportion of CD8+ LN T cells suggests that they can acquire CXCR3 after entering LNs and following their local activation by a specific antigen. It is indeed known that the great majority of $\mathrm{T}$ cells acquire CXCR3 in response to antigen activation, then recirculate, and are attracted by CXCR3 binding chemokines produced in inflamed tissues in response 
A
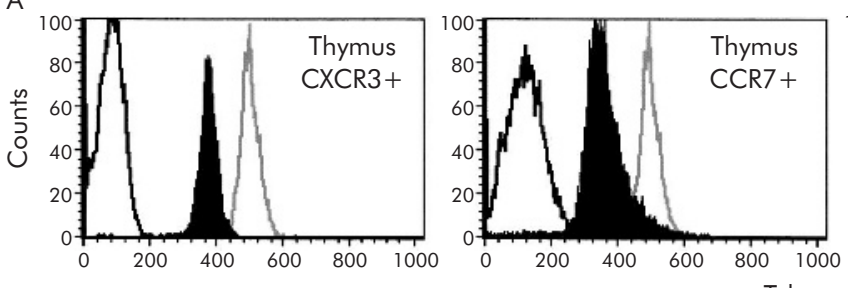

Telomere length
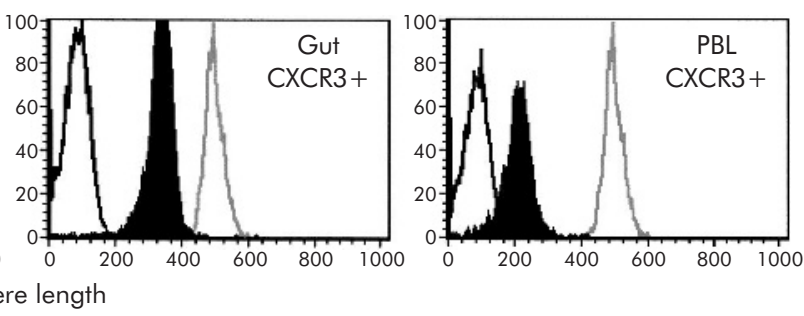

B
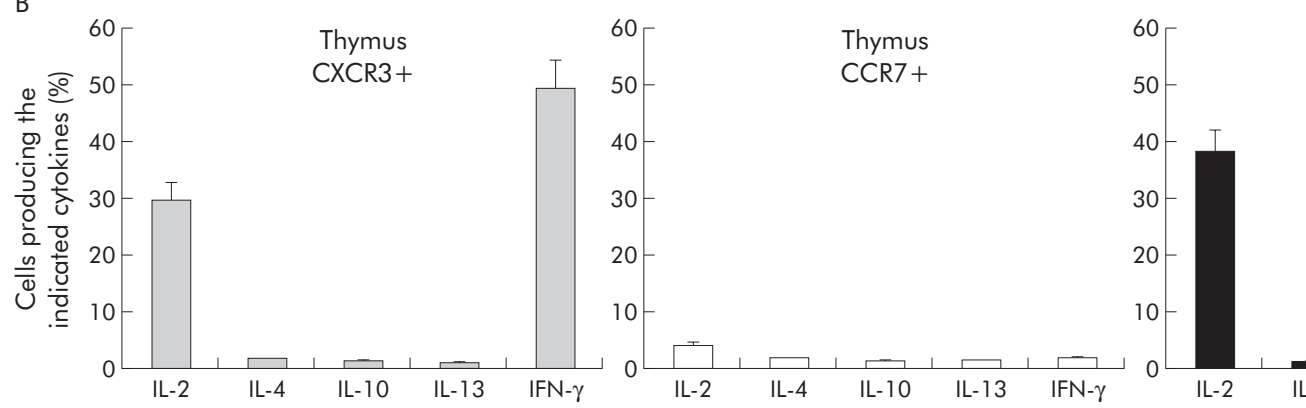

Gut

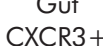

Figure 5 Assessment of telomere length and profile of cytokine production by the thymus, peripheral blood (PBL), or gut derived CD8+ T lymphocytes. (A) Telomere length of CD8+ cells from thymus (CXCR3+ and CCR7+), gut (CXCR3+), and PBL (CXCR3+). CD8+CXCR3+ and CD8+CCR7+ cells were positively selected by high gradient magnetic cell sorting from PB and gut mononuclear cell suspensions or thymocytes depleted from DP CD4+CD8+ cells, and then stained with a fluorescein conjugated telomere specific PNA probe, as described in material and methods. Cells of the tetraploid 1301 cell line were used as control cells having long telomeres. White histograms represent the unstained samples, black histograms the stained samples, and grey histograms the 1301 cell line. (B) Profile of cytokine production of CD8+ T cells from the thymus (CXCR3+ and CCR7+) and gut (CXCR3+), as assessed by intracellular detection by flow cytometry following polyclonal stimulation with PMA plus ionomycin. Columns represent mean (SEM) values of four separate experiments. IL, interleukin; IFN- $\gamma$, interferon $\gamma$.

to IFN- $\gamma$ and other cytokines. ${ }^{3}$ This can account for the presence of high numbers of CXCR3+CCR7+CD8+ T cells in PB. However, PB usually contained remarkable proportions of CD8+ $\mathrm{T}$ cells expressing CXCR3, but no CCR7. One explanation may be that these cells reflect the traffic into the circulation of CXCR3+CD8+ thymocytes. However, this possibility is unlikely as the majority of circulating CXCR3+CCR7-CD8+ $\mathrm{T}$ cells differed from CXCR3+CD8+ thymocytes because of the lack of $\mathrm{CDl} 03$ and a short telomere length compared with $\mathrm{T}$ cells which have undergone several cycles of cell division. ${ }^{25}{ }^{26}$ Thus it is more reasonable to believe that the majority of CXCR3+CCR7- CD8+ T cells present in adult $\mathrm{PB}$ are $\mathrm{T}$ lymphocytes activated in peripheral lymphoid tissues. ${ }^{27}$ Accordingly, we found that CCR7+CXCR3-CD8+ umbilical cord blood T cells could be induced to express CXCR3 after polyclonal stimulation in vitro and to lose CCR7, but not CXCR3, following prolonged activation (data not shown).

We also hypothesise that CXCR3+CD8+ thymocytes, because of their lack of CCR7, cannot enter peripheral lymphoid tissues through HEV, but migrate directly to gut mucosa where they colonise as IELs. A major role for CCR9 and its ligand CCL25 in the organisation of regional immunity at the level of the small intestinal compartment has been reported in both mice and humans. ${ }^{1421} 22$ Nevertheless, we found that CCR9 was expressed at the thymus level by CXCR3-CCR7+, but not by CXCR3+CCR7-, CD8+ $\mathrm{T}$ cell subset and was present in a small minority of human gut CXCR3+CD8+ IELs. Secondly, both thymic and circulating CD8+CXCR3+CD103+ $\mathrm{T}$ cells did not express CCR9. Thirdly, at least in our hands, the only gut IEL subset which consistently expressed CCR9 was represented by B lymphocytes. Thus the reasons for the discrepancies between these data and the results of previous studies ${ }^{142} 22$ remain unclear. On the other hand, the present results are consistent with those obtained in mice lacking CCR9, or in mice reconstituted with bone marrow derived haematopoietic progenitor cells that were transduced with a genetically modified CCL25 intrakine expressing gene and exhibit a reduction in TCR $\gamma \delta+$, but not TCR $\alpha \beta+$, gut IELs. ${ }^{29-31}$ This suggests a role for other chemokine receptors in the recruitment of the latter cell type. Accordingly, it has recently been shown that the majority of mouse TCR $\gamma \delta+$ cells express CCR9 whereas only a minority of CD8+ cells do. ${ }^{32}$ Moreover, in agreement with the finding reported here that CCR9 was expressed on intestinal B cells, it has recently been shown that the interaction between CCR9 and its ligand CCL25 is crucial for recruitment to, and permanence in, the intestinal mucosa of IgA secreting B lymphocytes. ${ }^{33} 34$ Another chemokine possibly involved in the migration of the CXCR3+CD8+ thymocyte subset to gut mucosa may be CXCL12, which has been found to be produced by intestinal epithelial cells. ${ }^{13}$ However, this possibility is unlikely, inasmuch as only a proportion of CXCR3+CD8+ gut T cells expressed CXCR4. The $\alpha_{\mathrm{E}} \beta_{7}$ integrin (identified by CD103) has been found to be essential for IEL localisation, ${ }^{35}$ inasmuch as it mediates adhesion of T cells to epithelial cells via their interaction with E-cadherin ${ }^{36-38}$ and to microvascular endothelial cells via the interaction with another still unknown ligand. ${ }^{39}$ Accordingly, epithelial cells from normal small bowel expressed high concentrations of both CXCL10 and E-cadherin, and in the same specimens CXCR3 was found to be localised mainly at the level of CD8+ IELs. Constitutive production of high amounts of both CXCL10 and CXCL9 by intestinal epithelial cells has been reported both in vivo ${ }^{40}$ and in vitro. ${ }^{41}$ Likewise, CXCR3 expression by virtually all fresh intestinal IELs has already been observed, ${ }^{39}$ thus supporting the view that interactions between CXCR3 and its ligands may play an essential role in the colonisation of CXCR3+ thymocytes to the gut mucosa.

CXCR3 expression by gut IELs had been attributed to chronic activation of these cells by luminal pathogens. ${ }^{39}$ The results of this study do not allow us to draw definitive conclusions on the naïve or memory effector phenotype of CXCR3+CD103+CD8+ T cells present in the gut. In agreement with the results of previous studies, showing that gut CD8+ 

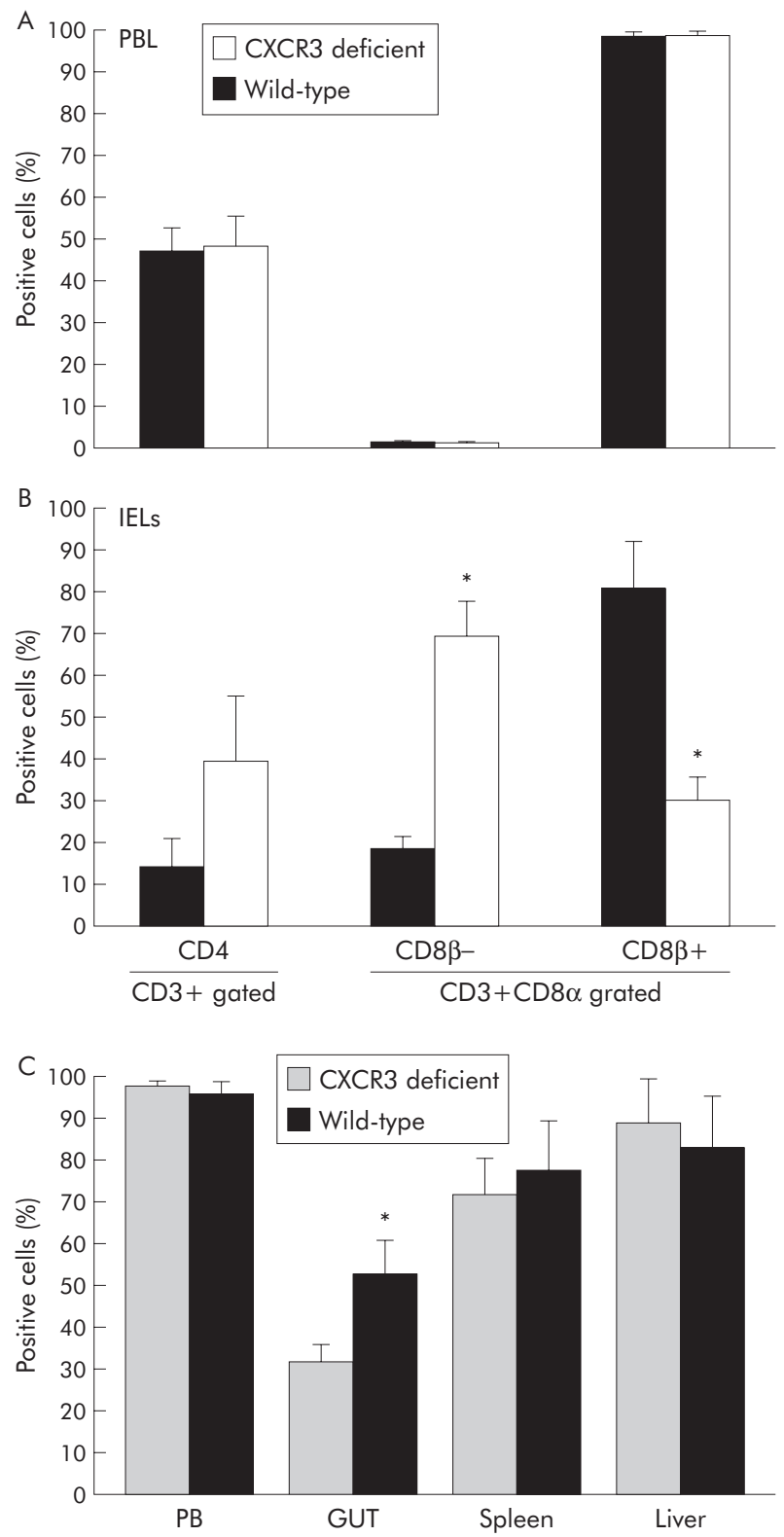

Figure 6 Reduced proportions of T cell receptor (TCR) $\alpha \beta+C D 8 \alpha \beta+T$ lymphocytes in the gut of CXCR3 gene deficient mice. (A) Percentages of $\mathrm{CD} 4, \mathrm{CD} 8 \alpha \alpha$, and CD8 $\alpha \beta$ T cells were evaluated in peripheral blood lymphocytes (PBL) of both CXCR3 deficient and wild-type mice.

(B) Percentages of CD4, CD $8 \alpha \alpha$, and CD $8 \alpha \beta$ T cells were evaluated in gut lymphocytes of both CXCR3 deficient and wild-type mice. Data represent mean (SD) obtained in four different experiments. IELs, intraepithelial lymphocytes. (C) Percentages of CD3+CD8 $\alpha \beta+T$ cells were evaluated in the indicated organs of CXCR3 deficient mice adoptively transferred with $C D 3+C D 8 \alpha \beta+$ thymocytes from wild-type mice or from non-treated CXCR3 deficient mice. Data represent mean (SD) values obtained in three different experiments. ${ }^{*} p<0.05$.

$\mathrm{T}$ cells prevalently exhibit a memory/effector phenotype, ${ }^{42-44}$ we found that both CXCR3+CD103+CD8+ T cells from the thymus and gut, unlike CCR7+CD8+ thymocytes, expressed activation markers, such as CD69 and CD45RO (data not shown). Furthermore, unlike CCR7+CD8+ thymocytes that do not produce any type of cytokine, CXCR3+CD8+ cells from both the thymus and gut showed the ability to produce IL-2 and IFN- $\gamma$ in response to polyclonal stimulation. On the other hand, analysis of the TCRV $\beta$ repertoire did not reveal enrichment for any V $\beta$ in gut IELs, as well as no significant differences not only between PB and gut lymphocytes but also between CXCR3+ and CCR7+ thymocytes, suggesting that no oligoclonal expansion due to repeated antigen stimulation had occurred (data not shown). Moreover, the great majority of intestinal CXCR3+CD8+ T cells exhibited a telomere length which was greater than that of CXCR3+CD8+ $\mathrm{PB} \mathrm{T}$ cells and rather comparable with CXCR3+CD8+, and more importantly with CCR7+CD8+, thymocytes, thus suggesting that even these cells had not undergone repeated cycles of cell division.

Strong support for the hypothesis of a major role of the interaction between CXCR3 and its ligands (mainly CXCL10) in the colonisation of CXCR3+CD8+ thymocytes to the gut mucosa was provided by experiments performed in CXCR3 deficient mice. These animals showed increased proportions of $\mathrm{CD} 3+\mathrm{CD} 8 \alpha \alpha+$ (which include both TCR $\alpha \beta$ and TCR $\gamma \delta$ cells), and decreased proportions of CD $3+C D 8 \alpha \beta+$ lymphocytes (which include only TCR $\alpha \beta$ cells) in the gut mucosa, but not in $\mathrm{PB}$, when compared with wild-type mice. Moreover, adoptive transfer of $\mathrm{CD} 3+\mathrm{CD} 8 \alpha \beta+$ thymocytes from wild-type into CXCR3 deficient mice resulted in a significant increase in CD3+CD8 $\alpha \beta+\mathrm{T}$ cells in the gut, but not in PB, spleen, or liver.

Thus taken together, these data suggest that CXCR3+CD103+ TCR $\alpha \beta+C D 8+$ SP thymocytes represent a distinct subset of $C D 8 \alpha \beta+\mathrm{T}$ cells, at least in part already equipped with the cytotoxic machinery, which may directly migrate in response to CXCR3 binding chemokines constitutively produced by epithelial cells into the gut mucosa where they probably play an important role as a first line of protection against infection and cell transformation.

\section{ACKNOWLEDGEMENTS}

We thank Dr Vittorio Vanini from the Apuanic Paediatric Hospital, Massa Carrara, Italy, Dr Renato Moretti and Dr Marco Farsi from the General Surgery Unit, Careggi Hospital, Florence, Italy, and Dr Geri Fratini from the Department of Physiopathology University of Florence, Italy for providing all human tissue specimens.

\section{Authors' affiliations}

F Annunziato, L Cosmi, F Liotta, E Lazzeri, P Romagnani, R Angeli,

L Lasagni, R Manetti, F Marra, I Petrai, F Tonelli, E Maggi,

S Romagnani, Centre for Research, Transfer, and High Education

"DENOthe", University of Florence, Florence, Italy

C Gerard, Ina Sue Perlmutter Laboratory, Children Hospital, Harvard Medical School, Boston, MA, USA

P Dello Sbarba, Department of Experimental Pathology and Oncology, University of Florence, Florence, Italy

This paper was supported by grants from Italian Ministry of University and Scientific Research, Italian Association for Cancer Research (AIRC), and by the EC.

Conflict of interest: None declared.

\section{REFERENCES}

1 Annunziato F, Romagnani $\mathrm{P}$, Cosmi L, et al. Chemokines and lymphopoiesis in human thymus. Trends Immunol 2001;22:277-81.

2 Butcher EC, Picker U. Lymphocyte homing and homeostasis. Science 1996:272:60-6.

3 Campbell JJ, Butcher EC. Chemokines in tissue-specific and microenvironment-specific lymphocyte homing. Curr Opin Immunol 2000;12:3336-414.

$4 \mathrm{Kim}$ CH, Pelus LM, White JR, et al. Differential chemotactic behavior of developing $\mathrm{T}$ cells in response to thymic chemokines. Blood 1998:91:4434-43.

5 von Adrian UH, Mackay CR. T-cell function and migration. N Engl J Med 2000;343:1020-34.

6 Girard JP, Springer TA. High endothelial venules (HEVs): specialized endothelium for lymphocyte migration. Immunol Today 1995; 16:449-57.

7 Campbell JJ, Hedrick J, Zlotnik MA, et al. Chemokines and the arrest of lymphocytes rolling under flow conditions. Science 1998;279:381-4.

8 Forster R, Schubel A, Breitfeld E, et al. CCR7 coordinates the primary immune response by establishing functional microenvironments in lymphoid organs. Cell 1999;99:23-33. 
9 Ngo VN, Tang HL, Cyster JC. Epstein-Barr virus-induced molecule 1 ligand chemokine is expressed by dendritic cells in lymphoid tissues and strongly attracts naïve T cells and activated B cells. J Exp Med 1998;188:181-91.

10 Gunn MD, Kyuwa S, Tam C, et al. Mice lacking expression of secondary lymphoid organ chemokine have defects on lymphocyte homing and dendritic cell localization. J Exp Med 1999; 189:451-60.

11 Annunziato F, Romagnani P, Cosmi L, et al. Macrophage-derived chemokine and EBI1-ligand chemokine attract human thymocytes in different stage of development and are produced by distinct subsets of medullary epithelial cells: possible implications for negative selection. J Immunol 2000;165:238-46.

12 Chantry D, Romagnani P, Raport CJ, et al. Macrophage-derived chemokine is localized to thymic medullary epithelial cells and is a chemoattractant for CD3+,CD4+,CD8low thymocytes. Blood 1999;94:1890-8.

13 Agace WW, Amara A, Roberts Al, et al. Constitutive expression of stromalderived factor 1 by mucosal epithelia and its role in HIV transmission and propagation. Curr Biol 2000; 10:325-8.

14 Zabel BA, Agace WW, Campbell JJ, et al. Human G protein-coupled GPR-9$6 / C C$ chemokine receptor 9 is selectively expressed on intestinal homing $T$ lymphocytes, mucosal lymphocytes, and thymocytes and is required for thymus-expressed chemokine-mediated chemotaxis. J Exp Med 1999; 190:1241-55

15 Vicari AP, Figueroa DJ, Hedrick JA, et al. TECK: a novel CC chemokine specifically expressed by thymic dendritic cells and potentially involved in T cell development. Immunity 1997; 7:291-301.

16 Phillips R, Ager A. Activation of pertussis toxin-sensitive CXCL12 (SDF-1) receptors mediates transendothelial migration of $T$ lymphocytes across lymph node high endothelial cells. Eur J Immunol 2002;32:837-47.

17 Romagnani $\mathbf{P}$, Annunziato F, Lazzeri E, et al. Interferon-inducible protein-10, monokine induced by interferon gamma and interferon-inducible T-cell alpha chemoattractant are produced by thymic epithelial cells and attract T-cell receptor (TCR) $\alpha \beta+$ CD $8+$ single-positive T cells TCR $\gamma \delta+$ T cells and natural killer-type cells in human thymus. Blood 2001;97:601-7.

18 Hancock WW, Lu B, Gao W, et al. Requirement of the chemokine receptor CXCR3 for acute allograft rejection. J Exp Med 2000;192:1515-20.

19 Annunziato F, Cosmi L, Galli G, et al. Assessment of chemokine receptor expression by human Th1 and Th2 cells in vitro and in vivo. J Leukoc Biol 1999:65:691-9.

20 Agace WW, Roberts Al, Wu L, et al. Human intestinal lamina propria and intraepithelial lymphocytes express receptors specific for chemokines induced by inflammation. Eur J Immunol 2000;30:819-26.

21 Kunkel EJ, Campbell JJ, Harldsen G, et al. Lymphocyte CC chemokine receptor 9 and epithelial thymus-expressed chemokine (TECK) expression distinguish the small intestine immune compartment: epithelial expression of tissue-specific chemokines as an organizing priniciple in regional immunity J Exp Med 2000;192:761-7.

22 Svensson M, Marsal J, Ericsson A, et al CCL25 mediates the localization of recently activated $C D 8$ alphabeta(+) lymphocytes to the small-intestinal mucosa. J Clin Invest 2002;110:1113-21.

23 Hayday A, Theodoridis E, Rambsburg E, et al. Intraepithelial lymphocytes: exploring the third way in immunology. Nat Immunol 2001;2:997-1003.

24 McFarland RD, Douek DC, Koup RA, et al. Identification of a human recen thymic emigrant phenotype. Proc Natl Acad Sci U S A 2000;97:4215-20.

25 Monteiro J, Batliwalla F, Ostrer H, et al. Shortened telomers in clonally expanded CD28- CD8+ T cells imply a replicative history that is distinct from their CD28+ CD8+ counterparts. J Immunol 1996;156:3587-90.
26 Batliwalla FM, Rufer N, Lansdorp PM, et al. Oligoclonal expansions in the CD8+CD28- T cells largely explain shorter telomers detected in the subset. Human Immunol 2000;61:951-8.

27 Sallusto $F$, Lenig $D$, Forster $R$, et al. Two subsets of memory $T$ lymphocytes with distinct homing potentials and effector functions. Nature 1999:401:708-12.

28 Champagne P, Ogg GS, King AS, et al. Skewed maturation of memory HIVspecific CD8 T lymphocytes. Nature 2001;410:106-11.

29 Wurbel MA, Malissen M, Guy-Grand D, et al. Mice lacking the CCR9 CC chemokine receptor show a mild impairment of early T- and B-cell development and a reduction in T-cell receptor $\gamma \delta+$ gut intraepithelial lymphocytes. Blood 2001;96:2626-32.

30 Uehara S, Grinberg A, Farber JM, et al. A role for CCR9 in T lymphocyte development and migration. J Immunol 2002;168:2811-19.

31 Onai N, Kitabatake M, Zhang YY, et al. Pivotal role of CCL25 (TECK)-CCR9 in the formation of gut cryptopatches and consequent appearance of intestinal intraepithelial T lymphocytes. Int Immunol 2002;14:687-94.

32 Uehara S, Song K, Farber JM, et al. Characterization of CCR9 expression and CCL25/thymus-expressed chemokine responsiveness during T cell development: CD3(high)CD69+ thymocytes and gammadeltaTCR+ thymocytes preferentially respond to CCL25. J Immunol 2002;168:134-42.

33 Bowman ED, Kuklin NA, Youngman KR, et al. The intestinal chemokine thymus-expressed chemokine (CCL25) attracts lgA antibody-secreting cells. $J$ Exp Med 2002; 195:269-75.

34 Pabst O, Ohl L, Wendland $M$, et al. Chemokine receptor CCR9 contributes to the localization of plasma cells to the small intestine. J Exp Med 2004; 199:411-16.

35 Schon MP, Arya A, Murphy EA, et al. Mucosal T lymphocyte numbers are selectively reduced in integrin $\alpha_{\mathrm{E}}$ (CD103)-deficient mice. J Immunol 1999:162:6641-9

36 Cepek KL, Parker CM, Madara JL, et al. Integrin $\alpha_{E} \beta_{7}$ mediates adhesion of T lymphocytes to epithelial cells. J Immunol 1993;150:3459-70.

37 Higgins JM, Mandelbrot DA, Shaw SK, et al. Direct and regulated interaction of integrin $\alpha_{E} \beta_{7}$ with E-cadherin. J Cell Biol 1998;140:197-210.

38 Taraszka K, Higgins JMG, Tan K, et al. Molecular basis for leukocyte integrin $\alpha_{E} \beta_{7}$ adhesion to epithelial (E)-cadherin. J Exp Med 2000;191:1555-67.

39 Strauch UG, Mueller RC, Li XY, et al. Integrin (CD103) mediates adhesion to intestinal microvascular endothelial cell lines via and $\mathrm{E}$-cadherin-independent interaction. J Immunol 2001;166:3506-14.

40 Dwinell MB, Lugering N, Eckmann L, et al. Regulated production of interferoninducible T-cell chemoattractants by human intestinal epithelial cells. Gastroenterology 2001;120:49-59.

41 Shibahara T, Wilcox JN, Madara JL. Characterization of epithelial chemoattractants for human intestinal intraepithelial lymphocytes. Gastroenterology 2001;120:60-70.

42 Lundqvist C, Melgar S, Yeung MM, et al. Intraepithelial lymphocytes in human gut have lytic potential and a cytokine profile that suggest $T$ helper 1 and cytotoxic functions. J Immunol 1996;157:1926-34.

43 Jabri B, de Serre NP, Cellier C, et al. Selective expansion of intraepithelia lymphocytes expressing the HLA-E-specific natural killer receptor CD94 in celiac disease. Gastroenterology 2000;1 18:867-79.

44 Roberts Al, Lee L, Schwarz E, et al. NKG2D receptors induced by IL-15 costimulate CD28-negative effector CTL in the tissue microenvironment. $\mathrm{J}$ Immunol 2001;167:5527-30. 